\title{
Energy Dispersive X-ray Spectroscopic Analysis of Al-Cu-Fe Quasicrystalline Thin Film Layer
}

\author{
Kira Smith ${ }^{1}$, Andrew Baker ${ }^{1}$, Jacob Beckey ${ }^{1}$, Chris Mankos ${ }^{1}$, and Chunfei $\mathrm{Li}^{1}$ \\ ${ }^{1}$ Clarion University, Clarion, PA 16214, USA
}

A quasicrystalline (QC) thin film in aluminum, copper, and iron (Al-Cu-Fe) alloy is reported. The Al-Cu$\mathrm{Fe}$ alloy is of interest due to its unique properties. Quasicrystals are known to be smooth and the metals used to synthesize the Al-Cu-Fe alloy are relatively cheap [1]. Recently, a QC thin film was discovered on the substrate of a column-like structure of $\lambda$ phase in the Al-Cu-Fe alloy, which has not been reported previously. Data collected through our recent studies provided a thickness measurement of the thin film. Our continued work will result in an estimated energy of incident electron beam to analyze the true composition of the thin film with minimum interference from the substrate.

The alloy was prepared by arc melting of pure $\mathrm{Al}, \mathrm{Cu}$, and Fe elements. A TESCAN Vega-3 XMU Scanning Electron Microscope (SEM) was used throughout our work. The thickness of the thin film was measured with an imaging software. The Monte Carlo Casino was researched for future use.

An SEM image of the column-like structure on the Al-Cu-Fe alloy is shown in Fig. 1(a). The arrow points to the location of column-like structure. Fig. 1(b) shows the thin film on the column-like structure. A portion of the thin film is enlarged and shown in the inlet. It was found that the thin film has a thickness of 142nm though ImageJ measurement on the SEM image, as shown in Fig. 2. Measurements were taken at optimum angles within the limit of tilt and rotation. The thickness of the thin film is not uniform over the entire column.

The ultimate goal of our future work is to find the composition of the thin film. The thickness will be used to determine the composition. Since the thickness is not uniform over the entire column, this will be taken into consideration when collecting data. The Monte Carlo Simulation will provide the optimum energy of the electron beam needed to penetrate the thin film. Fig. 3 shows examples of Monte Carlo Casino simulation to determine appropriate electron beam energy for thin film analysis. Monte Carlo Casino uses data of elements to generate x-ray, secondary, and backscattered electron signals in an SEM and shows how deep they penetrate at different beam energies [2]. The SEM will be used to perform Energy Dispersive X-ray Spectroscopy (EDS), which will allow us to find the true thin film composition.

\section{References}

[1] Goldman, A. I., \& Widom, M., 42 (1991), p. 685-729

[2] Hovington, Pierre, et al., 19 (1997), p. 1-14. 

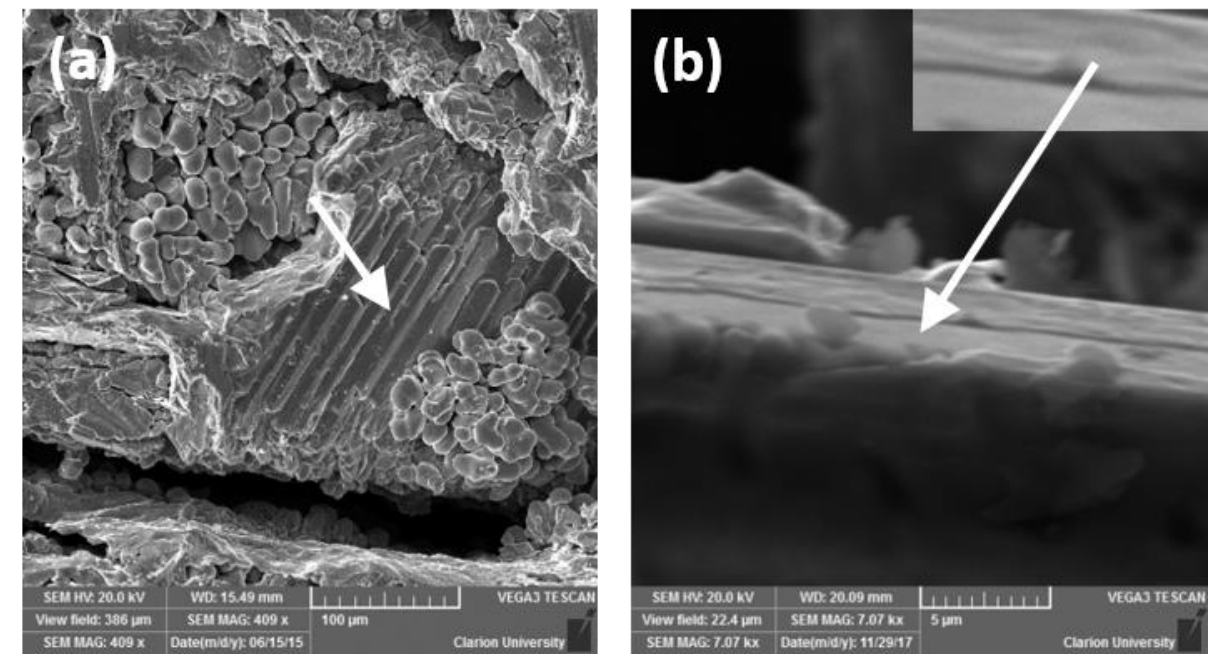

Figure 1. SEM images of column-like structure (a) and the thin film on column (b). The arrow in (a) marks the location of column-like structures and the inlet in (b) shows an enlarged area of the thin film.

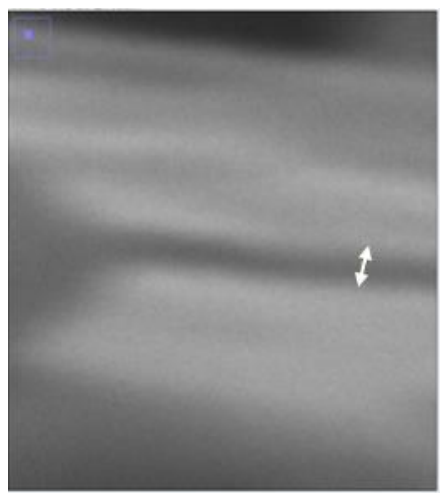

Figure 2. Measurement of film thickness based on the usage of ImageJ.

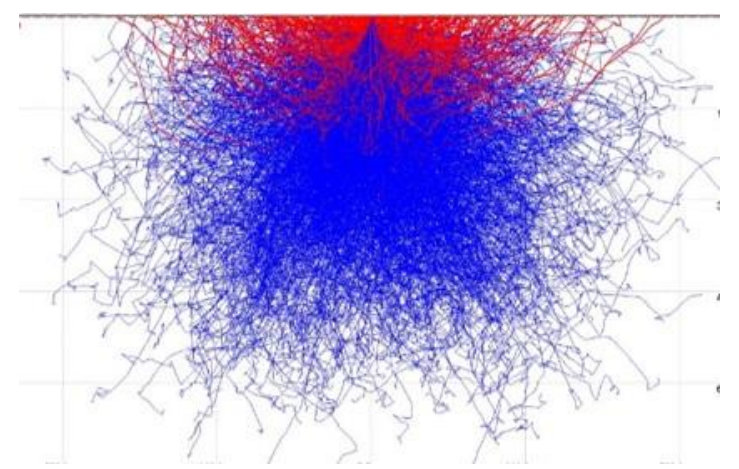

Figure 3. Example of Monte Carlo simulation. Red in a Casino MC plot represents backscattered electrons. 